\title{
The day after COVID-19 in IBD: how to go back to 'normal'
}

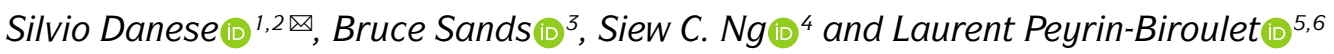

During the coronavirus disease 2019 (COVID-19) pandemic, inflammatory bowel disease (IBD) practices have been disrupted. This Comment summarizes the key strategies that should be implemented for both patients and IBD specialists to provide optimal care while avoiding new outbreaks for the first 6-12 months after the peak of the COVID-19 pandemic.

The WHO (World Health Organization) declared coronavirus disease 2019 (COVID-19), caused by the novel coronavirus SARS-CoV-2, a pandemic on 11 March $2020^{1}$. Globally, countries have adopted strict measures to contain the spread of SARS-CoV-2 (REF. $\left.{ }^{2}\right)$. Although cases are still increasing daily in some countries, in others, evidence suggests a 'flattening of the curve' and reduction in the incidence of infections.

No one can predict how things will evolve in the coming months, or when a return to some semblance of 'normal' activity might resume ${ }^{3}$. Several potential vaccines are currently under development ${ }^{4}$, but development is very slow, challenging and expensive, and the chances of producing an effective and scalable vaccine in the short term are low.

A study found that patients with inflammatory bowel disease (IBD) $(n=6,000)$ have no greater risk of COVID-19 infection than the healthy population ${ }^{5}$. Indeed, when infected, they developed less severe disease $^{5}$. However, during the pandemic, most elective outpatient visits, surgeries, endoscopies and infusions for patients with IBD have been postponed, and most patients are advised to maintain strict isolation. As we move back towards normality, clinicians need to recognize the best ways to care for patients with IBD. Here, we outline strategies for IBD management in the 6-12 months after the peak of the COVID-19 pandemic (TABLE 1).

\section{Education and psychological support}

As with all patients, those with IBD are advised to wear personal protective equipment (PPE), to adhere to proper social distancing procedures, to wash their hands properly with soap or alcohol-based sanitizer and to avoid touching their face ${ }^{6}$. Being isolated and unable to seek treatment during the pandemic might exacerbate the psychological symptoms of IBD. Thus, in the post-pandemic period, psychological or cognitive behavioural therapy should be offered to patients with IBD who display psychological symptoms and those awaiting surgery or endoscopy. In Wuhan, China, clinicians communicating with patients with IBD used social media (WeChat) extensively as an educational and instructional tool during the early stages of the pandemic, with excellent results ${ }^{7}$. During the post-pandemic period, these innovative methods should continue to be used to educate and instruct patients on best practices.

\section{Outpatient visits}

Before the pandemic, all patients were allowed an accompanying person at hospitals or clinics, and telemedicine was rarely used. However, during the pandemic, IBD specialists and nurses increased telemedicine use and rapidly implemented new ways of monitoring patients. If increased numbers of patients attend outpatient clinics in the post-pandemic period, priority should be given to patients with severe or uncontrolled IBD, or to newly diagnosed or referred patients. Telemedicine should continue to maintain contact and monitor patients who are not prioritized for outpatient visits, particularly patients in remission. Such patients should be advised to perform laboratory tests, such as for faecal calprotectin, close to home or use home-based tests if available.

\section{Infusions}

All infusions in the post-pandemic period should be administered according to routine clinical practice. A test for COVID-19 (if available) or a screening protocol with a phone or face-to-face interview should be implemented before admission to the clinic to systematically check for acute respiratory tract symptoms (dyspnoea, cough or fever) among patients with IBD, and no one should accompany them to the clinic. Patients who are SARS-CoV-2-negative or asymptomatic should receive intravenous infusions as planned, while those positive for SARS-CoV-2 or with highly suspicious COVID-19 symptoms should be referred to the COVID-19 clinic. During infusions, approximately $2 \mathrm{~m}$ between seats is considered safe, and no accompanying person should be allowed in the room. Rapid infusion protocols (30-60 min when possible) should be implemented, and when new subcutaneous biological agents are available, patients should be transitioned to subcutaneous 
Table 1 | Changes in IBD practice before, during and after the peak of the COVID-19 pandemic

\begin{tabular}{|c|c|c|c|}
\hline Service & Before & During & After \\
\hline Outpatient clinics & $\begin{array}{l}\text { All outpatient } \\
\text { consultations; telephone } \\
\text { and/or email helpline; } \\
\text { telemedicine infrequently } \\
\text { used; faecal calprotectin } \\
\text { tests mostly performed in } \\
\text { a laboratory; handwashing } \\
\text { before and after } \\
\text { examination }\end{array}$ & $\begin{array}{l}\text { Telemedicine for most patients (new and follow } \\
\text { up); telephone and/or email helpline; urgent } \\
\text { IBD clinic open; in many cases, laboratory tests } \\
\text { performed at nearest laboratory to avoid travel; } \\
\text { if available, faecal calprotectin home tests; use of } \\
\text { PPE for clinical staff and patients is mandatory; } \\
\text { room cleaning and air exchange between } \\
\text { patients; social distancing observed in waiting } \\
\text { room; patients should come unaccompanied }\end{array}$ & $\begin{array}{l}\text { Telemedicine for about one-third of patients } \\
\text { (follow up of sustained remission); telephone } \\
\text { and/or email helpline; use of PPE for clinical } \\
\text { staff and patients is mandatory; all faecal } \\
\text { calprotectin tests should be performed at } \\
\text { home to maintain a COVID-19-free clinic; } \\
\text { in many cases, laboratory tests performed } \\
\text { at nearest laboratory to avoid travel; social } \\
\text { distancing in waiting room; limit or avoid } \\
\text { accompanying persons }\end{array}$ \\
\hline \multirow[t]{3}{*}{ Endoscopy } & \multirow{3}{*}{$\begin{array}{l}\text { All endoscopic procedures; } \\
\text { gown, gloves; mask and } \\
\text { face shields often not worn }\end{array}$} & \multirow{2}{*}{$\begin{array}{l}\text { Urgent procedures: signs of moderate or severe } \\
\text { inflammation in new IBD diagnosis; severe flares; } \\
\text { bowel obstruction; jaundice in patients with } \\
\text { PSC-IBD }\end{array}$} & $\begin{array}{l}\text { Favour non-invasive monitoring tools for } \\
\text { treat-to-target strategy }\end{array}$ \\
\hline & & & \multirow{2}{*}{$\begin{array}{l}\text { Priority for endoscopic procedures (first } \\
3-6 \text { months after lockdown): mild-moderate } \\
\text { flares, surveillance for colorectal cancer and } \\
\text { postoperative recurrence, subacute bowel } \\
\text { obstruction, new IBD diagnosis with abnormal } \\
\text { biochemical test, symptomatic patients with } \\
\text { moderate or severe symptoms }\end{array}$} \\
\hline & & $\begin{array}{l}\text { COVID testing before procedures if available or } \\
\text { reimbursed; full PPE }\end{array}$ & \\
\hline \multirow[t]{2}{*}{ Infusion clinic } & \multirow{2}{*}{$\begin{array}{l}\text { All infusions; } \\
\text { accompanying persons } \\
\text { permitted; less than } \\
1-2 \text { m distance between } \\
\text { the seats }\end{array}$} & $\begin{array}{l}\text { All infusions; conversion of some patients to } \\
\text { home infusion if available }\end{array}$ & \multirow{2}{*}{$\begin{array}{l}\text { All infusions; reduction of waiting times; } \\
\text { distance between the seats should be more } \\
\text { than 1-2 m; use of PPE for clinical staff and } \\
\text { patients; as all biologics will be available as } \\
\text { subcutaneous maintenance, only intravenous } \\
\text { administration for induction should be } \\
\text { performed (where available); rapid infusion; } \\
\text { home infusion if safety, efficiency and logistics } \\
\text { are in place }\end{array}$} \\
\hline & & $\begin{array}{l}\text { Exclusion of COVID-19 red flags: checkpoints at } \\
\text { the hospital entrance; no accompanying person } \\
\text { permitted; verification of patient contacts; } \\
\text { rearrangement of seats respecting a distance of } \\
1-2 \text { m; use of PPE for clinical staff and patients }\end{array}$ & \\
\hline Surgery & All surgery & $\begin{array}{l}\text { Urgent surgery: oncological cases; severe } \\
\text { perianal disease or sepsis; acute severe } \\
\text { ulcerative colitis; symptomatic stricturing } \\
\text { Crohn's disease }\end{array}$ & $\begin{array}{l}\text { Favour urgent surgery (first } 3-6 \text { months after } \\
\text { lockdown); after 6-12 months, back } \\
\text { to normality? }\end{array}$ \\
\hline
\end{tabular}

IBD, inflammatory bowel disease; PPE, personal protective equipment; PSC, primary sclerosing cholangitis.

formulation. Also, implementation of home infusion, if available, should be considered to avoid travel to the hospital.

\section{Endoscopy}

During the pandemic, most endoscopies have been reduced and limited to urgent cases. Accumulating waiting lists should be progressively reduced by giving clinical judgment on the basis of priority. While the treat-to-target strategy has been one of the key indicators to monitor patients, non-invasive strategies should be favoured before going back to full normality. In the post-pandemic period, endoscopy should be considered in a coordinated and carefully planned way (Supplementary Box 1).

Local guidance should be followed with regard to pre-endoscopy testing for COVID-19. Although it is an imperfect strategy with possible errors owing to test sensitivity issues, some centres around the world have adopted nasopharyngeal swab PCR testing within $48 \mathrm{~h}$ before a planned procedure, enabling closer to normal throughput in the endoscopy suite, while maintaining full PPE for all procedures. Such measures, though imperfect, could be reassuring to patients who might be reluctant to return to hospitals or ambulatory endoscopy centres.

\section{Surgery}

After the COVID-19 pandemic, careful measures should be implemented to manage the potentially high number of patients needing surgery. Patients with IBD to potentially prioritize as surgical candidates include those with anorectal septic complications, advanced colorectal malignancy that might progress to unresectable disease, acute peritonitis, gastrointestinal haemorrhage, toxic megacolon, bowel obstruction, and who fail conservative treatment ${ }^{6}$. However, it should be analysed on an individual basis. Only essential staff should participate in the surgery ${ }^{8}$. Patients undergoing surgery should be tested for COVID-19 before the operation. Informed consent should be obtained, and consideration should be given to the possible risk of COVID-19 infection, expected length of stay and the possibility of surgical complications and other comorbidities ${ }^{6}$.

\section{Conclusions and post-pandemic strategy}

After the COVID-19 pandemic, there is a high likelihood of long clinical waiting lists owing to cancellations of surgeries and procedures during the pandemic. Efforts and plans must be geared towards ramping up the number of surgeries, infusions and daily endoscopic examinations in the post-pandemic period. The triage of patients according to an order of priority will be necessary. 
Before admission, patients should be instructed to make appointments online and undergo strict triage for COVID-19 symptoms, exposure history and the medical services required ${ }^{8}$. Patients with fever or respiratory symptoms, with or without a history of exposure to COVID-19, should be tested for SARS-CoV-2 following throat swab collection, chest CT scan and routine blood tests $^{8}$. In addition, patients who have a definitive exposure history, overseas travel history or signs of inflammation on chest CT images, and those in need of invasive procedures including chemotherapy, hospitalization, surgery and haemodialysis, should have throat swabs taken for SARS-CoV-2 real-time PCR testing, chest CT scan and routine blood tests if necessary ${ }^{8}$. Also, periodic local resurgences of COVID-19 are likely until definitive treatment or vaccination strategies are available, which might require nimble adjustments to scheduling and delivery of care, and resumption of the more restrictive practices that were needed during the initial pandemic.

The effectiveness of current antibody tests to diagnose active COVID-19 infections and determine whether patients with SARS-CoV-2-positive antibodies are protected from reinfection is unclear ${ }^{9,10}$. Patients with previously confirmed COVID-19 should be carefully assessed and retested to ensure that they are infection free before being allowed into the clinic. To ensure proper social distancing, clinic time should be allocated in 30-min blocks ${ }^{8}$. Only one patient should be permitted into the consulting room at any given time, and they should be attended by one physician in each consulting room. Patients should be mandated to wear face masks and to keep $2 \mathrm{~m}$ apart when waiting in line to prevent airborne transmission of SARS-CoV-2. For patients who need to be admitted to hospital, only they should be permitted into the ward ${ }^{8}$. After admission, only one household member should be allowed to take care of a critical patient, and both should be required to wear face masks during their time in the hospital and to fill in a COVID-19 questionnaire for secondary screening. Family members or next of kin should not be permitted for patients who are not in critical condition ${ }^{8}$.

For emergency patients without SARS-CoV-2 test results, meticulous protection protocols should be observed to ensure that life-saving operations are performed without nosocomial transmission of the virus. Non-emergency clinics for patients with IBD should take a careful and gradual approach in increasing the number of patients they see daily and should only operate at normal capacity once they have put everything in place to ensure the safety of patients and staff from potential COVID-19 infection.

These measures to help patients with IBD will probably only be necessary for the next 6-12 months if the promise of an effective vaccine is achieved within that time.

1. World Health Organization. WHO Director-General's opening remarks at the media briefing on COVID-19 - 11 March 2020 www.who.int https://www.who.int/dg/speeches/detail/who-directorgeneral-s-opening-remarks-at-the-media-briefing-on-covid-19--11 -march-2020 (2020).

2. Walensky, R. P. \& del Rio, C. From mitigation to containment of the covid-19 pandemic. JAMA 323, 1889-1890 (2020).

3. Fontanarosa, P. B. \& Bauchner, H. COVID-19-looking beyond tomorrow for health care and society. JAMA 323, 1907-1908 (2020).

4. Lurie., N., Saville, M., Hatchett, R. \& Halton, J. Developing Covid-19 vaccines at pandemic speed. N. Eng. J. Med. 382, 1969-1973 (2020).

5. Allocca, M. et al. Incidence and patterns of COVID-19 among inflammatory bowel disease patients from the Nancy and Milan cohorts. Clin. Gastroenterol. Hepatol. https://doi.org/10.1016/ j.cgh.2020.04.071 (2020).

6. Queiroz, N. S. F. et al. Management of inflammatory bowel disease patients in the COVID-19 pandemic era: a Brazilian tertiary referral center guidance. Clinics 75, e1909 (2020).

7. An, P. et al. Prevention of COVID-19 in patients with inflammatory bowel disease in Wuhan, China. Lancet Gastroenterol. Hepatol. 5, 525-527 (2020).

8. Han, J., Zhu, L., Wang, Y., Zeng, Z. \& Zhang, S. Resumption of daily services in a gastroenterology department in Guangzhou, China, in the wake of COVID-19. Lancet Gastroenterol. Hepatol. https:// doi.org/10.1016/S2468-1253(20)30133-3 (2020).

9. Abbasi, J. The promise and peril of antibody testing for COVID-19. JAMA 323, 1881-1883 (2020).

10. Alizargar, J. Risk of reactivation or reinfection of novel coronavirus (COVID-19). J. Formos. Med. Assoc. 119, 1123 (2020).

\section{Competing interests}

S.D. has served as a speaker, consultant and an advisory board member for Abbvie, Ferring, Hospira, Johnson \& Johnson, Merck, Millennium Takeda, Mundipharma, Pfizer, Tigenix, UCB Pharma and Vifor. B.S. has received financial support for research: Janssen, Takeda; consultancy: 4D Pharma, Abbvie, Allergan, Amgen, Arena Pharmaceuticals, Boehringer-Ingelheim, Capella Bioscience, Celgene, Celltrion Healthcare Co. Ltd., Ferring, Janssen, Lilly, Otsuka, Palatin Technologies, Pfizer, Progenity, Protagonist Therapeutics, Rheos Medicines, Salix Pharmaceuticals, Seres Therapeutics, Sienna Biopharmaceuticals, Takeda, Target PharmaSolutions, Therevance Biopharma R\&D and Vivelix Pharmaceuticals. S.C.N. has served as speaker for Janssen, Abbvie, Tillotts, Takeda, Olympus, Ferring. L.P.-B. has received consulting fees: Merck, AbbVie, Janssen, Genentech, Mitsubishi, Ferring, Norgine, Tillotts, Vifor, Therakos, Pharmacosmos, Pilège, BMS, UCB Pharma, Hospira, Celltrion, Takeda, Biogaran, Boerhinger Ingelheim, Lilly, Pfizer, HAC-Pharma, Index Pharmaceuticals, Amgen and Sandoz; lecture fees: Merck, AbbVie, Takeda, Janssen, Takeda, Ferring, Norgine, Tillots, Vifor, Therakos, Mitsubishi and HAC-Pharma.

Supplementary information

Supplementary information is available for this paper at https://doi.org/ 10.1038/s41575-020-0322-8. 